\title{
Qualidade de vida e custos diretos em pacientes com blefaroespasmo essencial e espasmo hemifacial, tratados com toxina botulínica-A
}

\author{
Quality oflife and direct cost regarding blepharospasm and hemifacial spasm patients \\ treated with botulinum toxin $A$
}

\author{
Midori Hentona Osaki ${ }^{1}$ \\ Rubens Belfort $\mathbf{J r}^{2}$
}

Trabalho de conclusão de curso apresentado à Universidade Federal de São Paulo para a obtenção do Título de Mestre Profissional em Administração da Prática Oftalmológica.

'Médica colaboradora do Setor de Plástica Ocular do Departamento de Oftalmologia da Universidade Federal de São Paulo - UNIFESP.

${ }^{2}$ Professor titular do Departamento de Oftalmologia da Universidade Federal de São Paulo - UNIFESP.

Endereço para correspondência: Midori Hentona Osaki, Rua Vergueiro, 2045/1009 - São Paulo (SP) CEP 04101-001

E-mail: midori_osaki@yahoo.com.br

Recebido para publicação em 22.11.2002

Versão revisada recebida em 27.02.2003

Aprovação em 09.05.2003

Nota Editorial: Pela análise deste trabalho e por sua anuência na divulgação desta nota, agradecemos aos Drs. Suzana Matayoshi e Carlos Ramos de Souza-Dias.

\begin{tabular}{|c|}
\hline RESUMO \\
\hline $\begin{array}{l}\text { Objetivo: Avaliar os custos e a qualidade de vida dos pacientes com } \\
\text { blefaroespasmo essencial e espasmo hemifacial, submetidos à terapia com } \\
\text { a toxina botulínica-A. Métodos: Vinte e oito pacientes, sendo } 16 \text { com blefa- } \\
\text { roespasmo essencial e } 12 \text { com espasmo hemifacial, tratados com toxina } \\
\text { botulínica-A, foram avaliados por meio de dois instrumentos: o SF-36 e a } \\
\text { Escala de Disfunção de Blefaroespasmo. Os custos foram calculados } \\
\text { segundo o número de unidades da toxina utilizadas por cada paciente no } \\
\text { período de umano. Resultados: O custo total diretofoi estimadoem } 1081,62 \\
\text { reais por ano ( } \pm 89,39 \text { ) parablefaroespasmo essencial e } 618,06 \text { reais por ano } \\
\text { ( } \pm 60,06) \text { para espasmohemifacial,para tratamento ambulatorial (p<0,001). } \\
\text { Antes do tratamento, todos os domínios do SF-36 foram significantemente } \\
\text { piores em ambas as doenças. Sob tratamento efetivo com toxina botulínica- } \\
\text { A, os aumentos nos índices do SF-36 evidenciaram mudanças nas taxas de } \\
\text { saúde geral da populaçãoem estudo, com diferença estatisticamente signifi- } \\
\text { cante entre as respostas pré e pós-tratamento (p<0,05). Relacionando-se o } \\
\text { SF-36 com Escala de Disfunção de Blefaroespasmo, foram observadas nos } \\
\text { casos de blefaroespasmo essencial, relações significantes positivas (capa- } \\
\text { cidade funcional, estado geral, aspecto emocional e aspectos sociais), ao } \\
\text { passo que nos pacientes com espasmo hemifacial não foi encontrada } \\
\text { nenhuma correlação. Conclusão: Este estudo demonstra notável impacto } \\
\text { negativo das doenças na qualidade de vida desses pacientes, e uma } \\
\text { significante melhora em ambas as doenças após tratamento com toxina } \\
\text { botulínica-A. Além disso, os dados fornecem subsídios parajustificaroalto } \\
\text { custo da aquisição da toxina botulínica-A, devido a seus consideráveis } \\
\text { benefícios para a qualidade de vida. }\end{array}$ \\
\hline
\end{tabular}

Descritores: Toxina botulínica tipo-A/uso terapêutico; Toxina botulínica tipo-A/economia; Espasmo hemifacial/quimioterapia; Blefaroespasmo/quimioterapia; Custos de cuidados de saúde; Qualidade de vida; Custos de medicamentos

\section{INTRODUÇÃO}

O blefaroespasmo essencial (BE) é uma doença idiopática que afeta o músculo orbicular oculi. Como conseqüência, observa-se o fechamento involuntário, espasmódico e bilateral das pálpebras. Este fechamento forçado pode evoluir para uma cegueira funcional com severa limitação social, pois interfere em atividades diárias como ler, caminhar, trabalhar e cuidar dos afazeres domésticos ${ }^{(1-6)}$. 
O espasmo hemifacial (EH) caracteriza-se por contração involuntária, na maioria das vezes unilateral, do sistema muscular inervado pelo nervo facial. Resulta da compressão desse nervo por um ramo anômalo da artéria basilar ou cerebelar. Acomete principalmente a região periorbital e espalha-se para os músculos da face (zigomático maior, zigomático menor, orbicular oris e mental) do mesmo lado. Os espasmos persistem durante o sono, ao contrário do que ocorre no $\mathrm{BE}^{(1,3,5-8)}$.

Segundo a literatura, a prevalência do BE é de 1,72 e de 7,4 a 14,5 para EH, para cada 100.000 indivíduos ${ }^{(9)}$.

A toxina botulínica é o tratamento de escolha para ambas as doenças ${ }^{(1-7)}$. Esta neurotoxina é produzida pela bactéria Clostridium botulinum e atua bloqueando a liberação da acetilcolina na junção neuro-muscular, levando à paralisia muscular, que permanece por vários meses, proporcionando benefícios clínicos significantes ${ }^{(10-1)}$. Esse efeito foi inicialmente observado em músculos extra-oculares de macacos ${ }^{(12)}$. A eficácia e a segurança da toxina botulínica do tipo-A (BTX-A) para o tratamento de $\mathrm{BE}$ e EH foram estabelecidas em vários estudos $^{(1-8)}$. Aproximadamente 76 a $90 \%$ desses pacientes beneficiam-se com as injeções da toxina e o enfraquecimento muscular inicia-se dois a sete dias após a injeção. Para os pacientes de BE, o efeito dura cerca de três meses ${ }^{(1-6)}$, em $90 \%$ dos casos. Os pacientes com EH experimentam um efeito mais prolongado, ao redor de 4 a 6 meses ${ }^{(1,3,5-8)}$.

Dos oito sorotipos antigenicamente distintos da toxina botulínica (A, B, C1, C2, D, E, F, G), sete são neurotoxinas. O tipo A é o mais potente e foi o primeiro a ser obtido de forma altamente purificada e estável. Está comercialmente disponível como Botox ${ }^{\circledR}$ (Allergan) e Dysport ${ }^{\circledast}$ (Biosintética). A potência clínica de uma unidade de Botox $^{\circledR}$ corresponde a aproximadamente quatro unidades de Dysport ${ }^{\circledR}$. A toxicidade é expressa em unidades. Uma unidade corresponde à dose letal média $\left(\mathrm{DL}_{50}\right)$ para um grupo de camundongos fêmeas SwissWebster, pesando cerca de 20 gramas. A dose letal média para o ser humano é estimada em 39U/ $\mathrm{kg}^{(2,10-11)}$. Em 1989, a droga foi liberada pelo FDA (Food and Drug Administration) para tratamento de estrabismo e blefaroespasmo essencial ${ }^{(11)}$.

Embora a maioria dos pacientes com BE e EH não apresente redução de expectativa de vida, freqüentemente sofrem de baixa auto-estima, depressão e interação social prejudicada ${ }^{(3,13)}$. Para determinar o impacto da doença sobre a qualidade de vida e a eficácia da BTX-A para estas doenças, questionários podem ser utilizados como o questionário de avaliação da qualidade de vida Medical Outcomes Study Short Form-36 Healthy Survey (SF-36) ${ }^{(14-15)}$ e a Escala de Disfunção de Blefaroespasmo $(\mathrm{EDB})^{(16-17)}$.

Os instrumentos de qualidade de vida têm adquirido reconhecimento como um importante instrumento para avaliar o impacto do tratamento na saúde e avaliar as percepções internas do ponto de vista do paciente. O SF-36 é um questionário genérico elaborado para avaliar a qualidade de vida, tendo sido validado no Brasil em 1997. Este instrumento mede dimensões relativas à saúde física e mental através de oito domínios: capacidade funcional, aspectos físicos, dor, estado geral de saú- de, vitalidade, aspectos sociais, aspectos emocionais e saúde mental (Figura 1). Seus resultados são medidos em escores de 0 (pior condição de saúde) a 100 (melhor condição de saúde).

A EDB, que mede a severidade da doença, avalia o impacto da doença nas atividades diárias dos pacientes. A EDB possui oito itens, com índices variando de 0 a 26 pontos.

\section{MÉTODOS}

Após obtenção do termo de consentimento foram avaliados 28 pacientes ( 12 homens e 16 mulheres), dos quais 16 eram portadores de BE, e 12 de EH. A idade média era de 66,4 anos (51 a 84 anos) para BE e 58,58 anos (38-71 anos) para EH. Todos os pacientes freqüentam o ambulatório de Plástica Ocular do Depto. de Oftalmologia da UNIFESP/EPM. O protocolo de pesquisa foi submetido e aferido pela Comissão de Ética em Pesquisa da UNIFESP/EPM (CEP $\left.{ }^{\circ}{ }^{\circ} 1000 / 02\right)$. Este estudo foi realizado em pacientes que já vinham sendo tratados com BTX-A por pelo menos um ano antes do início do estudo. Para garantir a confiabilidade dos dados, foram selecionados somente os pacientes cuja terapêutica já estava estabilizada, uma vez que ajustes na utilização da droga comprometeriam os resultados.

Foram excluídos os pacientes com outras desordens neurológicas e/ou psiquiátricas, assim como pacientes ainda não tratados com BTX-A.

Os pacientes receberam aplicação de BTX-A $\left(\right.$ Botox $^{\circledR} /$ Allergan Inc, Irvine, CA, USA), diluída em 2ml de solução salina sem conservante. As injeções foram aplicadas por via subcutânea, com uma agulha $30 \mathrm{G}$ e uma seringa de insulina. As doses de BTX-A foram individualizadas conforme dados constantes nas ficha clínica de cada paciente. A dose média foi de $50 \mathrm{U}$ ( 40 a 60U) para BE e de $35 \mathrm{U}$ ( 25 a $45 \mathrm{U}$ ) para EH. A dose utilizada foi de 2,5 a $5 \mathrm{U}$ por cada ponto de aplicação; na maioria dos pacientes foi de 2,5U. Nos pacientes com BE, a toxina foi aplicada em vários pontos nas pálpebras superiores, inferiores e região superciliar. Nos pacientes com EH, além dos pontos ao redor dos

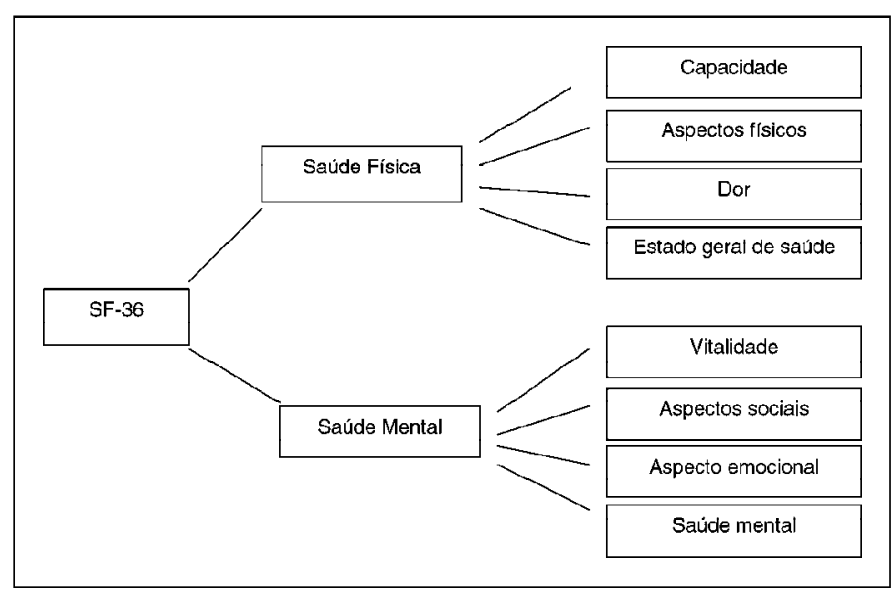

Figura 1 - Relações dos domínios do SF-36 


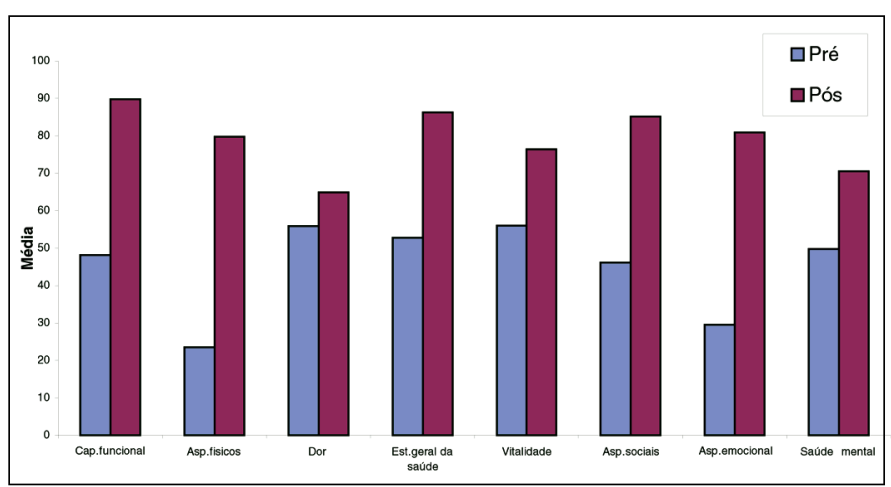

Figura 2 - Comparação dos índices do SF-36, pré e pós-tratamento em BE

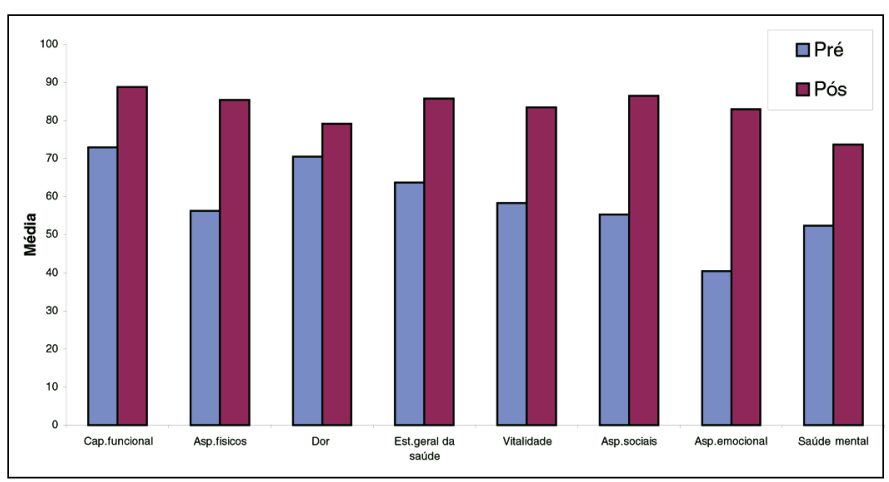

Figura 3 - Comparação dos índices do SF-36, pré e pós-tratamento em EH

olhos e região superciliar, foi aplicada em outros músculos do lado afetado. Em casos de assimetria, aplicamos pequenas quantidades da toxina na hemiface contralateral. Os pacientes foram orientados a utilizar colírios lubrificantes, para evitar a sensação de olho seco. O tempo médio da doença foi de 6,31 anos ( 1 a 12 anos) para BE, e de 3,50 anos ( 1 a 9 anos) para EH.

Em cada paciente, foram aplicados o SF-36 e a EDB, instantes antes da injeção da BTX-A e 4 semanas após ela. Com base na experiência anterior, escolheu-se esse período para avaliação por ser aquele em que os pacientes apresentam sua máxima resposta terapêutica. Para este estudo, foram excluídos dois itens: uso de óculos escuros e cinema, por não serem aplicáveis dadas às características da população estudada (aspectos culturais e /ou faixa etária).

Os custos diretos incluem, além do custo da BTX-A, os materiais utilizados para aplicação da toxina (agulha, seringa, gazes, luvas etc.) e as consultas. Estes foram estimados pelo período de um ano e calculados com base na tabela SUS. ${ }^{(18-19)}$ O custo da droga foi calculado de acordo com o total de doses durante o período de um ano. Para o cálculo das consultas, foi considerado que os pacientes com BE retornam ao ambulatório em torno de oito vezes ao ano e os pacientes com EH, seis vezes. Custos relativos à locomoção, outras drogas utilizadas para co-morbidade, gastos com acompanhantes e custos indiretos (como perda da produtividade decorrente da doença) não foram computados neste estudo.

Para realizar a análise estatística dos dados, foi adotado o teste de Wilcoxon, que respondeu pelas alterações nos valores do pré e pós-tratamento, na EDB e no SF-36. Para a correlação entre Escala de Incapacidade e pelo SF-36, no pré e no póstratamento, foi utilizado o coeficiente de correlação de Spearman. A comparação entre BE e EH foi efetuada através do teste não paramétrico de Mann-Whitney U. A variável idade média e tempo de doença foram comparados através do teste $t$.

\section{RESULTADOS}

Pacientes com BE apresentaram maior faixa etária e maior tempo de duração da doença, quando comparados com pacientes com EH. Utilizando-se o teste $t$, observamos diferenças estatisticamente significantes $(\mathrm{p}=0,002)$ para o variável tempo de doenças. Não foram encontradas diferenças significantes entre as idades médias no BE e EH $(\mathrm{p}=0,077)$. O custo total direto foi estimado em 1081,62 reais por ano $(\mathrm{DP} \pm 89,39)$ para $\mathrm{BE}$ e 618,06 reais por ano (DP $\pm 60,06)$ para $\mathrm{EH}$. Estes dados foram calculados estritamente para tratamento ambulatorial. Encontramos diferença estatisticamente significante $(\mathrm{p}<0,001)$ entre o custo total em $\mathrm{BE}$ e $\mathrm{EH}$ (teste não-paramétrico de Mann-Whitney). Os dados clínicos dos subgrupos estão resumidos na tabela 1 .

Os resultados da escala da severidade da doença, antes e depois da aplicação da BTX-A, estão resumidas na tabela 2.

A maioria dos pacientes apresentou de moderada a severa limitação antes do tratamento, pela EDB. Quanto maior o escore do EDB, pior a severidade da doença. Após o tratamento, todos os pacientes apresentaram melhora.

A média de índices para cada domínio de SF-36 no pré e pós-tratamento, em BE e EH, estão resumidos na tabela 3.

\begin{tabular}{|lcr|}
\hline & Tabela 1. Custos diretos e dados clínicos dos subgrupos & \\
Pacientes & Blefaroespasmo essencial & Espasmo hemifacial \\
Número & $\mathrm{N}=16$ & $\mathrm{~N}=12$ \\
Idade média (anos) & $66,44 \pm 10,94$ & $58,58 \pm 11,49$ \\
Sexo feminino \% & 75 & 50 \\
Tempo de doença (anos) & $6,31 \pm 2,86$ & $3,50 \pm 1,08$ \\
Custo anual do tratamento * (em reais) & $1081,62 \pm 89,39$ & $618,69 \pm 60,06$ \\
Teste $t$ & & \\
${ }^{*}$ Teste de Mann-Whitney & & \\
\hline
\end{tabular}




\begin{tabular}{|c|c|c|}
\hline & Pré & Pós \\
\hline $\mathrm{BE}$ & $16,06 \pm 2,26$ & $6,38 \pm 0,61$ \\
\hline $\mathrm{EH}$ & $12,33 \pm 1,72$ & $6,42 \pm 0,66$ \\
\hline \multicolumn{3}{|c|}{$\begin{array}{l}\text { BE=Blefaroespasmo essencial; EH=Espasmo hemifacial } \\
\text { Teste de Wilcoxon }\end{array}$} \\
\hline
\end{tabular}

Observou-se qualidade de vida significativamente inferior no BE em todos os domínios avaliados. Os piores resultados foram obtidos em relação a aspectos físicos e emocionais. Já no $\mathrm{EH}$, os piores resultados foram obtidos nas escalas de aspectos emocionais.

Sob tratamento efetivo com BTX-A, as mudanças nas taxas de saúde geral da população em estudo foram evidenciadas pelo aumento nos escores do SF-36, com diferença estatisticamente significante entre as respostas pré e pós-tratamento, para um nível de significância de 5\%, para ambas as doenças. Os níveis descritivos para os domínios do SF-36 estão descritos na tabela 4.

Foram excluídos dois itens: uso de óculos escuros e cinema. A Escala de Disfunção de Blefaroespasmo foi transformada (21- EDB original) de forma que quanto maior o valor, menor a severidade da doença.

Após os cálculos das correlações entre EDB e SF-36 (pelo método de Spearman), mostraram relações significantes positivas, nos casos de BE, a capacidade funcional, o estado geral, $\mathrm{o}$ aspecto emocional e os aspectos sociais. Já com os pacientes de EH, nenhuma correlação foi significante (ver Tabela 5).
Através do teste de Mann-Whitney U, as diferenças entre o pré e o pós-tratamento obtidas nos domínios do questionário SF-36 e na EDB, entre os grupos BE e EH, estão descritos na tabela 6 .

Para um nível de significância de 5\%, apenas os domínios capacidade funcional e aspectos físicos do SF-36 e a EDB apresentaram diferenças estatisticamente significante entre os grupos BE e EH. Nos três casos, as maiores diferenças entre os valores pré e pós-tratamento foram encontradas nos casos de BE.

\section{DISCUSSÃO}

O conceito de qualidade de vida tem sido considerado um importante instrumento para medir o impacto de doenças crônicas, como o BE e o EH ${ }^{(13,20-22)}$. Segundo a OMS, “a qualidade de vida é definida como percepção individual da posição do indivíduo na vida, no contexto de sua cultura e sistema de valores, nos quais ele está inserido, e em relação aos seus objetivos, expectativas, padrões e preocupações. É um conceito de alcance abrangente, afetado de forma complexa por sua saúde física, estado psicológico e nível de independência, por suas relações sociais e relações com as características do seu meio ambiente."

Em relação ao estudo da qualidade de vida e do impacto socioeconômico no BE e EH, poucos trabalhos da literatura internacional foram até hoje realizados e publicados ${ }^{(23-25)}$. O grau de sofrimento e de desamparo imposto pelos sintomas prejudica o desempenho desses pacientes em sua vida profissional, familiar e social.

\begin{tabular}{|c|c|c|c|c|}
\hline \multirow[b]{2}{*}{ Capacidade funcional } & BE pré & BE pós & HE pré & \multirow{2}{*}{$\begin{array}{c}\text { HE pós } \\
88,75 \pm 10,89\end{array}$} \\
\hline & $48,13 \pm 17,11$ & $89,69 \pm 9,56$ & $73,00 \pm 10,47$ & \\
\hline Aspectos fisicos & $23,44 \pm 23,21$ & $79,69 \pm 20,85$ & $56,25 \pm 26,38$ & $85,42 \pm 19,82$ \\
\hline Dor & $55,84 \pm 26,62$ & $64,91 \pm 21,76$ & $70,58 \pm 27,56$ & $79,17 \pm 25,91$ \\
\hline Estado geral da saúde & $52,81 \pm 21,38$ & $86,31 \pm 11,04$ & $63,75 \pm 12,49$ & $85,75 \pm 7,72$ \\
\hline Vitalidade & $55,94 \pm 3,30$ & $76,37 \pm 13,41$ & $58,33 \pm 15,27$ & $83,42 \pm 15,49$ \\
\hline Aspectos sociais & $46,09 \pm 6,00$ & $85,15 \pm 4,14$ & $55,29 \pm 14,60$ & $86,45 \pm 18,04$ \\
\hline Aspecto emocional & $29,56 \pm 23,66$ & $80,87 \pm 17,42$ & $40,42 \pm 28,07$ & $83,00 \pm 17,75$ \\
\hline Saúde mental & $49,75 \pm 3,88$ & $70,56 \pm 15,24$ & $52,33 \pm 4,93$ & $73,67 \pm 17,51$ \\
\hline
\end{tabular}

\begin{tabular}{|c|c|c|c|c|c|c|c|c|}
\hline & CF & AF & Dor & EG & VIT & AS & AE & SM \\
\hline $\begin{array}{l}\mathrm{BE} \\
p \text {-value }\end{array}$ & $<0,001$ & $<0,001$ & 0,044 & $<0,001$ & $<0,001$ & $<0,001$ & $<0,001$ & $<0,001$ \\
\hline $\begin{array}{l}\text { EH } \\
p \text {-value }\end{array}$ & 0,003 & 0,006 & 0,043 & 0,002 & 0,003 & 0,003 & 0,003 & 0,002 \\
\hline
\end{tabular}




\begin{tabular}{|c|c|c|c|c|c|c|c|c|c|}
\hline \multicolumn{2}{|l|}{ Domínio } & \multirow{2}{*}{$\begin{array}{c}\text { CF } \\
0,599\end{array}$} & \multirow{2}{*}{$\begin{array}{c}\text { AF } \\
0,161\end{array}$} & \multirow{2}{*}{$\begin{array}{c}\text { Dor } \\
-0,079\end{array}$} & \multirow{2}{*}{$\begin{array}{c}\text { EG } \\
0,598\end{array}$} & \multirow{2}{*}{$\begin{array}{c}\text { VIT } \\
-0,022\end{array}$} & \multirow{2}{*}{$\begin{array}{c}\text { AS } \\
0,605\end{array}$} & $\mathrm{AE}$ & SM \\
\hline \multirow{2}{*}{ EDB (transf) } & $\mathrm{BE}$ & & & & & & & 0,674 & 0,252 \\
\hline & $\mathrm{EH}$ & 0,481 & 0,379 & 0,045 & 0,162 & $-0,072$ & 0,252 & $-0,285$ & 0,325 \\
\hline
\end{tabular}

\begin{tabular}{|c|c|c|c|c|c|c|c|c|c|}
\hline \multicolumn{10}{|c|}{ Tabela 6. Comparação entre BE e EH, pré e pós BTX-A } \\
\hline Domínio & CF & AF & Dor & EG & VIT & AS & $\mathbf{A E}$ & SM & EDB \\
\hline$p$-value & 0,001 & 0,009 & 0,629 & 0,098 & 0,327 & 0,346 & 0,255 & 0,925 & $<0,001$ \\
\hline \multicolumn{10}{|c|}{ 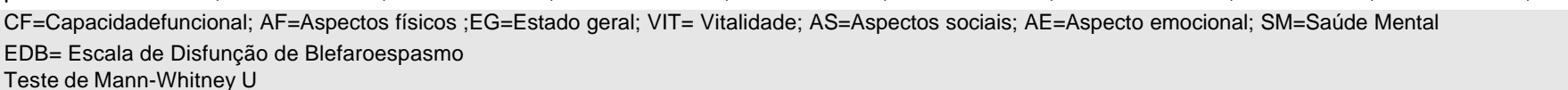 } \\
\hline
\end{tabular}

Na rotina clínica, a eficácia da terapêutica com BTX-A é usualmente avaliada através de resultados clínicos isolados, referentes aos aspectos físicos. Entretanto, o que importa efetivamente é saber, do ponto de vista da qualidade de vida, segundo o depoimento do paciente, se as mudanças desses índices representam melhoras reais dos prejuízos causados pela doença.

Neste estudo, nota-se um impacto negativo de ambas as doenças em vários domínios da SF-36 e uma significante melhora dos índices após o tratamento com a toxina. As limitações físicas e emocionais, assim como a diminuição da saúde mental, foram evidentes em pacientes de ambas as doenças.

Em geral, diante dos pacientes com $\mathrm{EH}$, os portadores de $\mathrm{BE}$ apresentaram maior incapacidade geral, com prejuízo da qualidade de vida. Entretanto, a maior média de idade dos pacientes com BE deve ser considerada na interpretação desses resultados. Os pacientes com BE apresentaram uma significante melhora dos aspectos físicos sob terapia com BTX-A. Entretanto, esse grupo já apresentava índices mais baixos no início do estudo, provavelmente devidos a maior grau de incapacidade relacionada à idade e co-morbidade.

Quanto aos aspectos emocionais, sociais e à saúde mental, há uma grande afluência de pacientes com BE que, anteriormente diagnosticados como pacientes psiquiátricos, são de fato pessoas comprometidas emocional e socialmente pela própria condição do quadro de BE, o que fica comprovado quando se registra a melhora de sua qualidade de vida após o tratamento com o BTX-A $\mathrm{A}^{(20-22,24-25)}$.

Os pacientes com BE severo experimentam forte restrição de suas capacidades funcionais, devido à limitação visual decorrente da doença. A cegueira parcial e o desconforto social causados pelo blefaroespasmo conduzem freqüentemente à ansiedade e à depressão, que não são sinônimos de distúrbios psicopatológicos ${ }^{(26-27)}$. Já o blefaroespasmo histérico, que aparece em pacientes mais jovens, melhora com a psicoterapia. É uma doença sem caráter evolutivo que não melhora com o repouso ou piora com o cansaço.
Resultados similares foram obtidos em estudos prévios, realizados em pacientes com distonia focal e não focal, utilizando-se dois questionários: SF-36 e EQ-5D (EuroQoL) antes e depois da aplicação de BTX-A. A severidade da doença foi medida através da escala de Tsui modificada (para a distonia cervical) e escala de movimento involuntário de blefaroespasmo (para a distonia cranial). Em ambas as doenças, foram observadas melhoras significantes em vários aspectos da qualidade de vida, após o tratamento com a BTX-A ${ }^{(22)}$. Em pacientes com distonia cervical tratado com a BTX-A, observou-se melhora da qualidade de vida em termos de dor, aspecto emocional e social, apesar do aumento do custo do tratamento com BTX-A ${ }^{(27)}$. Em casos de distonia cervical tratados com BTX-A foram observadas de baixas a médias correlações entre alterações clínicas, dor e incapacidade ${ }^{(23)}$. Outros autores relataram significante piora dos oito domínios do SF-36 nos pacientes com distonia cervical e BE, comparados com a população geral. Após terapia com BTX-A, houve melhora significante dos sintomas clínicos, mas não houve melhora na qualidade de vida nos pacientes com BE. Os pacientes com distonia cervical apresentaram melhora significante em apenas dois domínios do SF-36. Os autores enfatizam a necessidade de um suporte psicológico para tratar a depressão nesses pacientes ${ }^{(13)}$.

Em nosso estudo, os custos diretos do tratamento foram subestimados, já que este levantamento foi realizado numa instituição pública, onde os custos dos medicamentos são menores que os praticados no mercado (desconto institucional) e os custos relacionados com as consultas e os procedimentos seguiram os valores da tabela SUS ${ }^{(18-19)}$. Entretanto os custos relacionados ao número de consultas foram superestimados, pois foram realizados retornos após um mês, devido à necessidade de avaliar os efeitos da droga e aplicação dos questionários.

É preciso esclarecer que uma das peculiaridades dessa toxina é que, uma vez reconstituída, deve ser utilizada dentro de um período de quatro horas ${ }^{(13)}$. Além disso, para estimar o custo do tratamento para cada paciente, a quantidade da toxina foi calculada por unidades utilizadas, apesar de esta prática nem sempre ser possível. 
Segundo a OMS, em 2000, os gastos com a saúde no Brasil foram de 109 dólares por habitante ${ }^{(28)}$ e o custo do tratamento com a BTX-A foi de 320 e 570 dólares, respectivamente, para cada paciente com BE e EH.

As prevalências para BE e EH são de 1,72 e 7,4 a 14,5, respectivamente, para cada 100.000 indivíduos. Assim, se todos os pacientes com essas doenças no Brasil fossem tratados com BTX-A, o custo direto anual do tratamento seria de aproximadamente 10 a 17 milhões reais $(5,2$ a 8,9 milhões dólares, calculados em 2000).

\section{CONCLUSÕES}

Os dados confirmam que a BTX-A induz significante melhora temporária em vários aspectos da qualidade de vida em ambas as doenças. Além disso, os resultados podem contribuir para justificar os altos custos da terapia com a BTX-A, já que são consideráveis os benefícios para a qualidade de vida dos pacientes com BE e EH.

Estudos adicionais são necessários para elaboração de novos testes mais sensíveis às peculiaridades específicas das doenças crônicas como BE e EH e a mensuração do custo total.

\section{AGRADECIMENTOS}

À Dra. Massae Iwamura e à psicologa Ruth Nogueira da Silva Sobrinha pela grande ajuda na aplicação dos questionários.

\section{ABSTRACT}

Purpose: To evaluate both the costs and the impact on the quality of life of essential blepharospasm and hemifacial spasm patients treated with botulinum toxin-A. Methods: 28 patients (16 essential blepharospasm and 12 hemifacial spasm), treated with BTX-A, were evaluated by means of two research tools, namely Medical Outcomes Study Short Form Healthy Survey, and Fahn Disability Rating Scale. The costs were mainly calculated on the basis of the toxin units used by each patient during a one-year period. Results: The total estimated direct cost was estimated at $1,081.62$ reais per year $( \pm 89.39)$ for essential blepharospasm and 618.06 reais per year $( \pm 60.06)$ for hemifacial spasm, at an outpatient level $(\mathrm{p}<0.001)$. Before treatment all domains of SF-36 were significantly worse in both diseases. Under effective treatment with botulinum toxin-A the increases in the indices of SF-36 show changes in the general health rates of the population under study, with a statistically significant difference between the pre and post answers $(\mathrm{p}<0.05)$. In relating SF-36 with the Fahn Disability Rating Scale, the essential blepharospasm cases showed significant positive relationships (functional capacity, general health, emotional aspect and social aspects) while in the hemifacial spasm patients no correlation was found. Conclusion: The present study demonstrates a remarkable negative impact of both diseases on the quality of life of essential blepharos- pasm and hemifacial spasm patients, as well as a significant improvement of both diseases after botulinum toxin-A treatment. Furthermore, the research data seem to justify the high costs of botulinum toxin-A treatment in view of its considerable benefits regarding the patients' quality of life.

Keywords: Botulinum toxin type A/therapeutic use; Botulinum toxin type A/economics; Hemifacial spasm/drug therapy; Blepharospasm/therapy/drug therapy; Health care costs; Life of quality; Drug costs

\section{REFERÊNCIAS}

1. Jost WH, Kohl A. Botulinum toxin: evidence-based medicine criteria in blefarospasm and hemifacial spasm. J Neurol 2001;248(supl1):21-4.

2. Scott AB, Kennedy RA, Stubbs HA. Botulinum A toxin injection as a treatment for blepharospasm. Arch Ophthalmol 1985;103:347-50.

3. Ainsworth JR, Kraft SP. Long-term changes in duration of relief with botulinum toxin treatment of essencial blepharospasm and hemifacial spasm. Ophthalmology 1995;102:2036-40.

4. Jankovic J, Schwartz KS. Longitudinal experience with botulinum toxin injections for treatment of blepharospasm and cervical dystonia. Neurology. 1993;4:834-6.

5. Barbosa ER, Haddad MS, Silva HCA, Carvalho MJ, Bittar MS. Espasmo hemifacial: tratamento com toxina botulínica. Arq Bras Neurocir 1994;13:119-22.

6. Andrade LA, Borges V, Ferraz HB, Azevedo-Silva SM. Experiência com aplicação de toxina botulínica A em 115 pacientes. Arq Neuropsiquiatr 1997; 55(3B):553-7.

7. Borodic GE. Hemifacial spasm: evaluation and management, with emphasis on botulinum toxin therapy. In: Jankovic J, Hallett M, editors. Neurological disease and therapy. Therapy with botulinum toxin. New York: Marcel Dekker: 1994. p.331-51.

8. Cunha MC, Aguirre OP, Dias CRS. Tratamento do espasmo facial unilateral com toxina botulínica tipo A. Arq Bras Oftalmol 1998;61:54-60.

9. Dodel RC, Kirchner A, Koehne-Volland R, Künig G, Ceballos-Baumann A, Naumann M, et al. Costs of treating dystonias and hemifacial spasm with botulinum toxin A. Pharmacoeconomics 1997;12:695-706.

10. Bell MS, Vermeulen, LC Sperling KB. Pharmacotherapy with botulinum toxin: harnessing nature's most potent neurotoxin. Pharmacotherapy 2000;20:1079-91.

11. Huang, W, Jill A, Rogachefsky, A. Pharmacology of botulinum toxin. J Am Acad Dermatol 2000;43:249-59.

12. Scott AB, Rosenbaum A, Collins CC. Pharmacologic weakening of extraocular muscles. Invest Ophthalmol 1973;12:924-7.

13. Müller J, Kemmler G, Wissel J, Schneider A, Voller B, Grossmann J, et al. The impact of blepharospasm and cervical dystonia on health-related quality of life and depression. J Neurol 2002;249:842-6.

14. Ware JE, Snow KK, Kosinski M. Scoring the SF-36. SF-36 health survey manual and interpretation guide. Boston, MA: The Health Institute, New England Medical Center; 1993.

15. Ciconelli RM. Tradução para o português e validação do questionário de avaliação de qualidade de vida "Medical outcomes study 36 -item short-form healty survey (SF-36)" [tese]. S.Paulo: Universidade Federal de S. Paulo; 1997.

16. Fahn S. The assessment of the primary dystonias. In: Munsat: TL, editor. The quantification of neurologic deficit. Boston: Butterworth;1989. p.242-5.

17. Lindeboom R, De Haan R, Aramidesh M, Speelman JD. The blepharospasm disability scale: an instrument for the assessment of functional health in blepharospasm. Mov Disord 1995;10:444-9.

18. Secretaria de Estado da Saúde de São Paulo. Ata de registro de preços. [online]. (citado 2003 Jun 27]. Disponível em URL: http:/www.farma.saude. sp.gov.br/ata/SsaudeTMedic.asp

19. Brasil. Ministério da Saúde. DATASUS. Gasto médio (SUS) por atendimento ambulatorial [online]. [citado 2003 set 27]. Disponível em URL: http:// tabnet.datasus.gov.br/cgi/idb2001/e10.pdf

20. Tucha O, Naumann M, Alders GL, Lange KW. Quality of life in patients with blepharospasm. Acta Neurol Scand 2001;103:49-52.

21. Hilker R, Schischniaschvili M, Ghaemi M, Jacobs A, Rudolf J. Health related quality of life is improved by botulinum neurotoxin type A in long term treated patients with focal dystonia. J Neurol Neurosurg Psychiatry 2001;71:193-5 
22. Gudex CM, Hawthorne MR, Butler AG, Duffey P. Effect of dystonia and botulinum toxin treatment on health-related quality of life. Mov Disord 1998;13:941-9.

23. Brefel-Courbon C, Simonetta-Moreau M, Moré C, Rascol O, Clanet M, Montastruc JL, et al. A pharmacoeconomia evaluation of botulinum toxin in the treatment of spasmodic torticollis. Clin Neuropharmacol 2000;23:203-7.

24. Gudex CM, Hawthorne MR, Butler AG, Duffey PO. Measuring patient benefit from botulinum toxin in the treatment of dystonia. Feasibility of cost-utility analysis. Pharmacoeconomics. 1997;12:675-84.

25. Schnider P, Birner P, Moraru E, Auff E. Long-term treatment with botulinum toxin: dosage, treatment schedules and costs. Wien Klin Wochenschr 1999;111:59-65.
26. Wenzel T, Schnider P, Gringl H, Birner P, Nepp J, Auff E. Psychiatric disorders in patient with blepharospasm - a reactive pattern? J Psych Res 2000;48:589-91.

27. Scheidt CE, Schuller B, Rayki O, Kommerell G, Deuschl G. Relative absence of psychopathology in bening essential blepharospasm and hemifacial spasm. Neurology 1996;47:43-5.

28. World Health Organization. Comparison on selected indicator WHO Region Brazil. (Comparaed with other countries in WHO Region of the Americas) Indicator: Per capita government expenditure on health at average exchange rate (US\$), 2000. [online]. [citado 2003 set 27]. Genebra, WHO. Available from URL: http://www3.who.int/whosis/country/compare.cfm?language= en\&country $=$ bra\&indicator $=$ strPcGovEOHinUSD 2000

\section{Congresso da Sociedade Brasileira de Administração em Offalmologia - SBAO}

\section{0 e 11 de Junho de 2004 Centro de Convenções Ribalta - RJ}

INFORMAçÕES: Tel./Fax: (1 1 ) 3266-4538

E-mail:sbao@sbao.com.br

Home-page: www.oftalmo.org.br 